\title{
PARAMETRIZATION FOR SOME BOUNDARY VALUE PROBLEMS OF INTERPOLATION TYPE
}

\author{
Miklós Rontó - NATAlia Shchobak
}

\begin{abstract}
We obtain some results concerning the investigation of two-dimensional non-linear boundary value problems of interpolation type. We show that it is useful to reduce the given boundary value problem, using an appropriate substitution, to a parametrized boundary value problem containing some unknown scalar parameter in the boundary conditions. To study the transformed parametrized problem, we use a method which is based upon special types of successive approximations constructed in an analytic form.
\end{abstract}

\section{Introduction}

There are various methods aimed at the investigation of boundary value problems for ordinary differential equations. In [1, 2, 3, 4, 4, 5], various iteration schemes are used. The works [6, 7, 8, 9, 10, 11] develop the method of averaging of functional corrections and other projection-iterative methods. Topological methods based upon the application of degree theory are used in [12, 13, 14] in studies of the solvability of boundary value problems.

Numerical methods for various two-point and multipoint boundary value problems are dealt with in [15, 16, 17] and in the works cited therein. The numerical investigation of boundary value problems is usually based on the shooting method. In this case, as a rule, one does not deal with the situation where the values of the unknown solution are confined within a given bounded set. This may cause certain difficulties because the regularity conditions for the right-hand side (in particular, the Lipschitz condition) should be assumed globally, i. e., for all the values of the space variables. Complications of this kind can be overcome in some cases by using the numerical-analytic approach.

2000 Mathematics Subject Classification: 34B15, $34 \mathrm{~B} 08$.

Keywords: parametrized boundary-value problems, interpolation conditions, numericalanalytic method of successive approximations.

The first author was partially supported by the Hungarian National Research Foundation OTKA through Grant No. K68311. 


\section{MIKLÓS RONTÓ - NATALIA SHCHOBAK}

Amongst the wide variety of methods available for studying boundary value problems, the so-called numerical-analytic methods, which are based upon successive approximations, belong to the few of them that offer constructive possibilities for the investigation of the existence and the construction of the approximate solution for periodic, two-point, multipoint, parametrized boundary value problems [18, 19, 20, 21, 22, 23, 24, 25, 26, 27, 28].

According to the basic idea of the numerical-analytic methods, the given boundary value problem is reduced to some "perturbed" boundary value problem containing an unknown vector parameter. The solution of the modified problem is sought for in an analytic form by successive iterations. As for the way how the modified problem is constructed, it is essential that the form of the "perturbation term", which depending on the original differential equation and boundary condition, yields a certain system of algebraic or transcendental "determining equations", which determine the numerical values for the initial values of the solutions and the values of the parameters. By studying these determining equations, one can establish existence of results for the original boundary value problem.

Our aim is to show that, for some types of nonlinear boundary problems, it is useful to introduce certain parametrization techniques. In particular, this allows one to avoid dealing with singular matrices in the boundary condition and to simplify the construction of the successive approximation of the solution in an analytic form.

\section{Problem setting}

Let us consider the system of nonlinear differential equations

$$
x^{\prime}(t)=f(t, x(t)), \quad t \in[0, T]
$$

with the conditions of interpolation type

$$
\begin{gathered}
x_{1}(0)=x_{10}, \\
x_{1}(T)=x_{1 T},
\end{gathered}
$$

where $x=\operatorname{col}\left(x_{1}, x_{2}\right):[0, T] \rightarrow \mathbb{R}^{2}, f:[0, T] \times D \rightarrow \mathbb{R}^{2}$ is continuous and $D \subset \mathbb{R}^{2}$ is a closed and bounded domain. The boundary conditions (2) can be rewritten in the matrix form

$$
A x(0)+C_{1} x(T)=d,
$$

where $A=\left(\begin{array}{ll}1 & 0 \\ 0 & 0\end{array}\right), C_{1}=\left(\begin{array}{ll}1 & 0 \\ 0 & 0\end{array}\right)$, and $d=\left(\begin{array}{c}x_{10}+x_{1 T} \\ 0\end{array}\right)$. The matrix $C_{1}$ is obviously singular. 


\section{Parametrization of the boundary conditions}

To work around the singularity of the matrix $C_{1}$, we replace the value of the second component of the solution (11), (3) at a point $T$ by a parameter $\lambda$ :

$$
x_{2}(T)=\lambda \text {. }
$$

Using the relation (44), the boundary condition (3) can be rewritten as

$$
A x(0)+C x(T)=d(\lambda),
$$

where $C=\left(\begin{array}{ll}1 & 0 \\ 0 & 1\end{array}\right)$ and $d(\lambda)=\left(\begin{array}{c}x_{10}+x_{1 T} \\ \lambda\end{array}\right)$. Here, in contrast to $C_{1}$ in (3), the matrix $C$ is nonsingular.

Remark 1. The original non-linear problem of interpolation type (11), (2) is equivalent to the boundary-value problem (11) with boundary conditions (5).

We shall show that the transformed boundary value problem (11), (5) can be investigated by using some techniques based upon successive approximations. For any $x=\operatorname{col}\left(x_{1}, x_{2}\right) \in \mathbb{R}^{2}$, we use the notation $|x|=\operatorname{col}\left(\left|x_{1}\right|,\left|x_{2}\right|\right)$ and understand the inequalities between the 2 -dimensional vectors component-wise.

\section{Construction of the successive approximations}

We first introduce some notation.

Notation 1. If $\beta: I \rightarrow \mathbb{R}^{2}$ is a function, $I \subset \mathbb{R}$, and $z \in \mathbb{R}^{2}$, then $B_{I}(z, \beta)$ denotes the set

$$
B_{I}(z, \beta):=\left\{x \in \mathbb{R}^{2}:|x-z| \leq \beta(\lambda) \text { for any } \lambda \in I\right\} .
$$

Notation 2. If $D \subset \mathbb{R}^{2}$ is a set and $f:[0, T] \times D \rightarrow \mathbb{R}$ is a continuous function, then

$$
\delta_{D}(f):=\frac{1}{2}\left[\max _{(t, x) \in[0, T] \times D} f(t, x)-\min _{(t, x) \in[0, T] \times D} f(t, x)\right] .
$$

In (6), the min and max operations are understood in the componentwise sense.

We suppose in the sequel that the following conditions hold for the boundary value problem (1), (2).

(A) The function $f$ is continuous on $[0, T] \times D$ and, for a certain non-negative constant matrix $K=\left(K_{i j}\right)_{i, j=1}^{2}$, satisfies the Lipschitz condition of the form

$$
|f(t, x)-f(t, y)| \leq K|x-y|,
$$

for all $t \in[0, T]$ and $\{x, y\} \subset D$. 


\section{MIKLÓS RONTÓ - NATALIA SHCHOBAK}

(B) The set

$$
D_{\beta}:=\left\{z \in D: B_{I}(z, \beta(z, \cdot)) \subset D\right\}
$$

is non-empty, where $I:=\left\{\lambda \in \mathbb{R}:\left(\begin{array}{c}x_{1 T} \\ \lambda\end{array}\right) \in D\right\}$ and

$$
\beta(z, \lambda):=\frac{T}{2} \delta_{D}(f)+|[d(\lambda)-(A+E) z]|,
$$

where $E$ is the unit matrix.

(C) The greatest eigenvalue, $\lambda_{\max }(K)$, of $K$ satisfies the inequality

$$
\lambda_{\max }(K)<\frac{10}{3 T} .
$$

Let us define the set $U \subset \mathbb{R}$ as follows:

$$
U:=\left\{u \in \mathbb{R}: \operatorname{col}\left(x_{10}, u\right) \in D_{\beta}\right\} .
$$

With the given problem we associate the sequence of functions $\left\{x_{m}(\cdot, u, \lambda)\right\}$ given by the formula

$$
\begin{aligned}
x_{m}(t, u, \lambda):=z+ & \int_{0}^{t} f\left(s, x_{m-1}(s, u, \lambda)\right) \mathrm{d} s \\
& \quad-\frac{t}{T} \int_{0}^{T} f\left(s, x_{m-1}(s, u, \lambda)\right) \mathrm{d} s+\frac{t}{T}[d(\lambda)-(A+E) z],
\end{aligned}
$$

where $m=1,2,3, \ldots, x_{0}(t, u, \lambda)=\operatorname{col}\left(x_{10}, u\right)=: z$, and $u \in U$.

The values of $x_{m}(\cdot, u, \lambda)$ at the points $t=0$ and $t=T$ satisfy the equalities

$$
x_{m}(0, u, \lambda)=z
$$

and

$$
x_{m}(T, u, \lambda)=z+d(\lambda)-(A+E) z .
$$

So functions (10) satisfy the boundary conditions (2) for any $u \in U$ and $\lambda \in I$.

The following statement establishes the convergence of the sequence (10) and its relation to the transformed boundary value problem (1), (44), (15).

Theorem 1. Assume that $f:[0, T] \times D \rightarrow \mathbb{R}^{2}$ satisfies conditions (A)-(C). Then:

(I) The sequence (10) converges to the function $x^{*}(t, u, \lambda)$ as $m \rightarrow \infty$, uniformly in $(t, u, \lambda) \in[0, T] \times U \times I$.

(II) The limit function

$$
x^{*}(t, u, \lambda):=\lim _{m \rightarrow \infty} x_{m}(t, u, \lambda)
$$


is the unique solution of the integral equation

$$
x(t)=z+\int_{0}^{t} f(s, x(s)) \mathrm{d} s-\frac{t}{T}\left[\int_{0}^{T} f(s, x(s)) \mathrm{d} s+[d(\lambda)-(A+E) z]\right],
$$

i.e., a solution of the problem

$$
\begin{gathered}
x^{\prime}(t)=f(t, x(t))+\Delta(u, \lambda), \quad t \in[0, T], \\
A x(0)+C x(T)=d(\lambda),
\end{gathered}
$$

with the initial value at $t=0$ equal to $x^{*}(0, u, \lambda)=z=\operatorname{col}\left(x_{10}, u\right)$. Here,

$$
\Delta(u, \lambda):=-\frac{1}{T} \int_{0}^{T} f\left(s, x^{*}(s, u, \lambda)\right) \mathrm{d} s+\frac{1}{T}[d(\lambda)-(A+E) z] .
$$

(III) The following error estimate holds:

$$
\left|x^{*}(t, u, \lambda)-x_{m}(t, u, \lambda)\right| \leq \frac{20}{9} t\left(1-\frac{1}{T}\right) Q^{m-1}(E-Q)^{-1} h,
$$

where

$$
Q=\frac{3}{10} T K
$$

and

$$
h:=\delta_{D}(f) Q+K|d(\lambda)-(A+E) z| .
$$

Proof. We shall prove that, under the conditions assumed, sequence (10) is a Cauchy sequence in the Banach space $C\left([0, T], \mathbb{R}^{2}\right)$ equipped with the usual uniform norm. First we show that $x_{m}(t, u, \lambda) \in D$ for all $(t, u, \lambda) \in[0, T] \times U \times I$ and $m \in \mathbb{N}$. Indeed, using the estimate [23, Lemma 4]

$$
\left|\int_{0}^{t}\left[f(\tau)-\frac{1}{T} \int_{0}^{T} f(s) \mathrm{d} s\right] \mathrm{d} \tau\right| \leq \frac{1}{2} \alpha_{1}(t)\left[\max _{t \in[0, T]} f(t)-\min _{t \in[0, T]} f(t)\right]
$$

where

$$
\alpha_{1}(t)=2 t\left(1-\frac{t}{T}\right), \quad\left|\alpha_{1}(t)\right| \leq \frac{T}{2}, \quad t \in[0, T]
$$

relation (10) for $m=0$ implies that

$$
\begin{aligned}
\left|x_{1}(t, u, \lambda)-z\right| & \leq\left|\int_{0}^{t}\left[f(t, z)-\frac{1}{T} \int_{0}^{T} f(t, z) \mathrm{d} s\right] \mathrm{d} t\right|+|d(\lambda)-(A+E) z| \\
& \leq \alpha_{1}(t) \delta_{D}(f)+\tilde{\beta}(z, \lambda) \leq \beta(z, \lambda),
\end{aligned}
$$




\section{MIKLÓS RONTÓ - NATALIA SHCHOBAK}

where

$$
\tilde{\beta}(z, \lambda)=|d(\lambda)-(A+E) z|
$$

Therefore, by virtue of (8), we conclude that $x_{1}(t, u, \lambda) \in D$ whenever $(t, u, \lambda) \in$ $[0, T] \times U \times I$. By induction, one can easily show that all functions (10) are also contained in the domain $D$ for all $m=1,2, \ldots, t \in[0, T], u \in U, \lambda \in I$.

Consider the difference of functions

$$
\begin{aligned}
x_{m+1}(t, u, \lambda)-x_{m}(t, u, \lambda)= & \int_{0}^{t}\left[f\left(s, x_{m}(s, u, \lambda)\right)-f\left(s, x_{m-1}(s, u, \lambda)\right)\right] \mathrm{d} s \\
& -\frac{t}{T} \int_{0}^{t}\left[f\left(s, x_{m}(s, u, \lambda)\right)-f\left(s, x_{m-1}(s, u, \lambda)\right)\right] \mathrm{d} s
\end{aligned}
$$

and introduce the notation

$$
d_{m}(t, u, \lambda):=\left|x_{m}(t, u, \lambda)-x_{m-1}(t, u, \lambda)\right|, \quad m=1,2, \ldots
$$

Using the estimate from [21, Lemma 2.3] and taking the Lipschitz condition into account, we get

$$
d_{m+1}(t, u, \lambda) \leq K\left[\left(1-\frac{t}{T}\right) \int_{0}^{t} d_{m}(s, u, \lambda) \mathrm{d} s+\frac{t}{T} \int_{t}^{T} d_{m}(s, u, \lambda) \mathrm{d} s\right]
$$

for every $m=0,1,2, \ldots$ According to (17), we have

$$
d_{1}(t, u, \lambda)=\left|x_{1}(t, u, \lambda)-z\right| \leq \alpha_{1}(t) \delta_{D}(f)+\tilde{\beta}(z, \lambda)
$$

where $\tilde{\beta}(z, \lambda)$ is given by (18). Now we need the estimate

$$
\alpha_{m+1}(t) \leq\left(\frac{3}{10} T\right) \alpha_{m}(t) \leq\left(\frac{3}{10} T\right)^{m} \bar{\alpha}_{1}(t), \quad t \in[a, b], \quad m \geq 0,
$$

obtained in [21, Lemma 2.4] for the sequence of functions

$$
\alpha_{m+1}(t)=\left(1-\frac{t}{T}\right) \int_{0}^{t} \alpha_{m}(s) \mathrm{d} s+\frac{t}{T} \int_{t}^{T} \alpha_{m}(s) \mathrm{d} s, \quad m=0,1,2, \ldots
$$


where $\alpha_{0}(t)=1, \alpha_{1}(t)=2 t\left(1-\frac{t}{T}\right)$, and $\bar{\alpha}_{1}(t)=\frac{10}{9} \alpha_{1}(t)$. In view of (22) , (24), it follows from (21) for $m=1$ that

$$
\begin{aligned}
d_{2}(t, u, \lambda) \leq & K \delta_{D}(f)\left[\left(1-\frac{t}{T}\right) \int_{0}^{t} \alpha_{1}(s) \mathrm{d} s+\frac{t}{T} \int_{t}^{T} \alpha_{1}(s) \mathrm{d} s\right] \\
& +K \tilde{\beta}(z, \lambda)\left[\left(1-\frac{t}{T}\right) \int_{0}^{t} \mathrm{~d} s+\frac{t}{T} \int_{t}^{T} \mathrm{~d} s\right] \\
\leq & K\left[\alpha_{2}(t) \delta_{D}(f)+\alpha_{1}(t) \tilde{\beta}(z, \lambda)\right] .
\end{aligned}
$$

By induction, we can easily obtain

$$
d_{m+1}(t, u, \lambda) \leq K^{m}\left[\alpha_{m+1}(t) \delta_{D}(f)+\alpha_{m}(t) \tilde{\beta}(z, \lambda)\right], \quad m=0,1,2, \ldots,
$$

where $\alpha_{m+1}$ and $\alpha_{m}$ are computed according to (24) and $\delta_{D}(f), \tilde{\beta}(z, \lambda)$ are given by (6) and (8). By virtue of the second estimate in (23), relation (25) yields

$$
\begin{aligned}
d_{m+1}(t, u, \lambda) & \leq \bar{\alpha}_{1}(t)\left[\left(\frac{3}{10} T K\right)^{m} \delta_{D}(f)+K\left(\frac{3}{10} T K\right)^{m-1} \tilde{\beta}(z, \lambda)\right] \\
& =\bar{\alpha}_{1}(t)\left[Q^{m} \delta_{D}(f)+K Q^{m-1} \tilde{\beta}(z, \lambda)\right]
\end{aligned}
$$

for all $m=1,2, \ldots$, where the matrix $Q$ is given by formula (16). Therefore, in view of (26), we have

$$
\begin{aligned}
& \left|x_{m+j}(t, u, \lambda)-x_{m}(t, u, \lambda)\right| \\
& \leq\left|x_{m+j}(t, u, \lambda)-x_{m+j-1}(t, u, \lambda)\right| \\
& \quad+\left|x_{m+j-1}(t, u, \lambda)-x_{m+j-2}(t, u, \lambda)\right|+\cdots+\left|x_{m+1}(t, u, \lambda)-x_{m}(t, u, \lambda)\right| \\
& =\sum_{i=1}^{j} d_{m+i}(t, u, \lambda) \\
& \leq \bar{\alpha}_{1}(t)\left[\sum_{i=1}^{j}\left(Q^{m+i} \delta_{D}(f)+K Q^{m+i-1} \tilde{\beta}(z, \lambda)\right)\right] \\
& =\bar{\alpha}_{1}(t)\left[Q^{m} \sum_{i=0}^{j-1} Q^{i} \delta_{D}(f)+K Q^{m-1} \sum_{i=0}^{j-1} Q^{i} \tilde{\beta}(z, \lambda)\right]
\end{aligned}
$$

Since, due to conditions (C), the greatest eigenvalue of the matrix $Q$ of the form (16) does not exceed the unity, we have

$$
\sum_{i=0}^{j-1} Q^{i} \leq(E-Q)^{-1} \quad \text { and } \quad \lim _{m \rightarrow \infty} Q^{m}=0 .
$$




\section{MIKLÓS RONTÓ - NATALIA SHCHOBAK}

Therefore, we can conclude from (27) that, according to the Cauchy criterion, the sequence $x_{m}(t, u, \lambda)$ of the form (10) uniformly converges in the domain $(t, u, \lambda) \in[0, T] \times U \times I$. Since all functions $x_{m}(t, u, \lambda)$ of the sequence (10) satisfy the boundary condition (5) the limit function $x^{*}(t, u, \lambda)$ also satisfies these conditions. Passing to the limit as $m \rightarrow \infty$ in equality (10), we show that the limit function satisfies both the integral equation (12) and the integro-differential equation (13). Estimate (15) is an immediate consequence of (27).

Let us formulate the following statement concerning a control parameter. Consider the Cauchy problem

$$
\begin{aligned}
& x^{\prime}(t)=f(t, x(t))+\mu, \quad t \in[0, T], \\
& x(0)=z=\operatorname{col}\left(x_{10}, u\right),
\end{aligned}
$$

where $z \in D_{\beta}$ and $\mu \in \mathbb{R}^{2}$ is control parameter.

TheOREM 2. Under the conditions of Theorem 1, the solution $x=x(t, 0, z)$ of the initial value problem (28), (29) satisfies the boundary conditions (5) if and only if

$$
\mu=\Delta(u, \lambda),
$$

where $\Delta: U \times I \rightarrow \mathbb{R}^{2}$ is the mapping defined by the formula (14).

P r o of. According to the Picard-Lindelöf existence theorem, it is easy to show that the Lipschitz condition (17) implies that the initial value problem (28), (29) has a unique solution for all $(\mu, u) \in \mathbb{R}^{2} \times U$. It follows from the proof of Theorem 1 that, for every fixed

$$
(u, \lambda) \in U \times I,
$$

the limit function (11) of the sequence (10) satisfies the integral equation (12) and, in addition, $x^{*}(t, u, \lambda)=\lim _{m \rightarrow \infty} x_{m}(t, u, \lambda)$ satisfies the boundary condition (44). This implies immediately that the function $x=x^{*}(t, w, u, \lambda)$ of the form (11) is the unique solution of the initial value problem

$$
\begin{gathered}
\frac{d x(t)}{\mathrm{d} t}=f(t, x(t))+\Delta(u, \lambda), \quad t \in[0, T], \\
x(0)=z,
\end{gathered}
$$

where $\Delta(w, u, \lambda)$ is given by (14). Hence, (32), (33) coincides with (28), (29) corresponding to

$$
\mu=\Delta(u, \lambda)=-\frac{1}{T} \int_{0}^{T} f(s, x(s)) \mathrm{d} s+\frac{1}{T}[d(\lambda)-(A+E) z] .
$$

The fact that the function (11) is not a solution of (28), (29) for any other value of $\mu$, not equal to (34), is obvious from equation (301). 
The following statement explains the relation of the limit function $x=x^{*}(t, u, \lambda)$ to the solution of the transformed parametrized boundary value problem (11), (5).

Theorem 3. Let conditions $(\mathrm{A})-(\mathrm{C})$ be satisfied. Then the pair $\left(x^{*}\left(\cdot, u^{*}, \lambda^{*}\right), \lambda^{*}\right)$ is a solution of the transformed boundary value problem (1), (5) with a parameter $\lambda$ if and only if the pair $\left(u^{*}, \lambda^{*}\right)$ satisfies the system of the determining equations

$$
\Delta(u, \lambda)=-\frac{1}{T} \int_{0}^{T} f\left(s, x^{*}(s, u, \lambda)\right) \mathrm{d} s+\frac{1}{T}[d(\lambda)-(A+E) z]=0 .
$$

P r o of. It suffices to apply Theorem 2 and notice that the differential equation in (32) coincides with (11) if and only if the pair $\left(u^{*}, \lambda^{*}\right)$ satisfies the equation

$$
\Delta\left(u^{*}, \lambda^{*}\right)=0
$$

i. e., when the relation (35) holds.

In practice, it is natural to fix some natural $m$ and, instead of the exact determining system (35) to consider the approximate determining system

$$
\Delta_{m}(u, \lambda)=-\frac{1}{T} \int_{0}^{T} f\left(s, x_{m}(s, u, \lambda)\right) \mathrm{d} s+\frac{1}{T}[d(\lambda)-(A+E) z]=0,
$$

whence, taking into account condition (4), we find approximate values of the unknown parameters $(u, \lambda) \in U \times I$.

\section{An application}

Consider the system

$$
\begin{aligned}
& x_{1}^{\prime}(t)=x_{2}(t), \\
& x_{2}^{\prime}(t)=\frac{t^{2}}{50}-\frac{t}{4} x_{2}^{2}(t)-\frac{t}{10} x_{1}(t),
\end{aligned}
$$

where $t \in[0,1]$, with the boundary conditions

$$
x_{1}(0)=-\frac{1}{10}, \quad x_{1}(1)=\frac{1}{10} .
$$

It is easy to check that the following vector-function is an exact solution of the above problem:

$$
x_{1}(t)=\frac{t}{5}-\frac{1}{10}, \quad x_{2}(t)=\frac{1}{5} .
$$




\section{MIKLÓS RONTÓ - NATALIA SHCHOBAK}

Suppose that the boundary value problem is considered in the domain

$$
D:=\left\{\left(x_{1}, x_{2}\right):\left|x_{1}\right| \leq 0.5,\left|x_{2}\right| \leq 0.5\right\} .
$$

The boundary condition (37) can be rewritten in the form

$$
A x(0)+C_{1} x(T)=d,
$$

where $A=\left(\begin{array}{ll}1 & 0 \\ 0 & 0\end{array}\right), C_{1}=\left(\begin{array}{ll}1 & 0 \\ 0 & 0\end{array}\right)$ and $d=\left(\begin{array}{l}0 \\ 0\end{array}\right)$. Here $C_{1}$ is a singular matrix.

Let us replace the value of the second component of the solution of the boundary value problem (36), (39) at a point $T$ by a parameter $\lambda$ :

$$
x_{2}(T)=\lambda,
$$

where $\lambda \in[-0.5,0.5]$. Using (40), the boundary condition (39) can be rewritten as

$$
\left(\begin{array}{ll}
1 & 0 \\
0 & 0
\end{array}\right) x(0)+\left(\begin{array}{ll}
1 & 0 \\
0 & 1
\end{array}\right) x(T)=\left(\begin{array}{l}
0 \\
\lambda
\end{array}\right)
$$

where $C=E$ is a nonsingular matrix.

One can verify that, for the boundary value problem (36)-(41), conditions (A)-(C) are fulfilled in the domain $D$ with the matrices

$$
A:=\left[\begin{array}{ll}
1 & 0 \\
0 & 0
\end{array}\right], \quad C:=\left[\begin{array}{ll}
1 & 0 \\
0 & 1
\end{array}\right], \quad K:=\left[\begin{array}{cc}
0 & 1 \\
\frac{1}{10} & \frac{1}{4}
\end{array}\right] .
$$

Indeed, from the Perron theorem it is known that the greatest eigenvalue $\lambda_{\max }(K)$ of the matrix $K$ in virtue of the nonnegativity of its elements is real, nonnegative and computations show that

$$
\lambda_{\max }(K) \leq 0.47 \text {. }
$$

Moreover, the vectors $\delta_{D}(f)$ and $\beta(z, \lambda)$ in (9) are such that

$$
\begin{gathered}
\delta_{D}(f) \leq\left[\begin{array}{c}
\frac{1}{2} \\
\frac{13}{160}
\end{array}\right], \\
\beta(z, \lambda):=\frac{T}{2} \delta_{D}(f)+|d(\lambda)-(A+E) z| \leq\left[\begin{array}{c}
\frac{9}{20} \\
\frac{13}{320}
\end{array}\right]+\left|\begin{array}{c}
0 \\
\lambda-u
\end{array}\right| .
\end{gathered}
$$

Thus, we can proceed with application of the numerical-analytic scheme described above and thus construct the sequence of approximate solutions.

The result of the first iteration is

$$
\begin{aligned}
& x_{11}(t, u, \lambda)=\frac{t}{5}-\frac{1}{10}, \\
& x_{12}(t, u, \lambda)=\frac{t^{3}}{150}-\frac{t^{2} u^{2}}{8}+\frac{t^{2}}{200}-\frac{7 t}{600}+\frac{t u^{2}}{8}+t \lambda-u t+u
\end{aligned}
$$

for all $t \in[0,1], u \in U, \lambda \in[-0.5,0.5]$. 


\section{PARAMETRIZATION FOR SOME BVPS OF INTERPOLATION TYPE}

The computation shows that the approximate solutions of the first approximate determining equation are $u=0.2, \lambda=0.2066666667$. The first and the second components of the first approximation are

$$
x_{11}(t)=\frac{t}{5}-\frac{1}{10}, \quad x_{12}(t)=0.006666666667 t^{3}+0.2 .
$$

The result of the second iteration is

$$
\begin{aligned}
x_{21}(t, u, \lambda)= & \frac{t^{4}}{600}-\frac{t^{3} u^{2}}{24}+\frac{t^{3}}{600}-\frac{7 t^{2}}{1200}+\frac{t^{2} u^{2}}{16}+\frac{t^{2} \lambda}{2}-\frac{t^{2} u}{2}+\frac{u t}{2}+\frac{81 t}{400} \\
& -\frac{t u^{2}}{48}-\frac{t \lambda}{2}-\frac{1}{10}
\end{aligned}
$$

and

$$
\begin{aligned}
x_{22}(t, u, \lambda)= & \frac{t^{2}}{200}-\frac{t^{4} \lambda u^{2}}{64}+\frac{t^{4} \lambda u}{8}+\frac{7 u t^{3}}{3600}-\frac{u t^{3} \lambda}{6}+\frac{t \lambda u}{24}-\frac{49 t^{4}}{5760000}-\frac{t^{6} u^{2}}{57600} \\
& +\frac{t^{3} u^{2}}{6}+\frac{t^{7} u^{2}}{16800}-\frac{t^{5} u}{6000}-\frac{t^{5} u^{2}}{4800}+\frac{t^{5} u^{4}}{640}-\frac{t^{5} \lambda}{2000}-\frac{t^{5} u^{3}}{80}+\frac{t^{4} u^{3}}{32} \\
& -\frac{t^{4} u}{480}-\frac{2393 t^{4} u^{2}}{38400}+\frac{7 t^{4} \lambda}{4800}-\frac{t^{4} u^{4}}{1024}-\frac{t^{4} \lambda^{2}}{16}-\frac{u^{3} t^{3}}{48}+\frac{t u^{3}}{480}+\frac{t \lambda^{2}}{16} \\
& +\frac{t u^{4}}{15360}-\frac{t^{6} \lambda}{1800}+\frac{t^{6} u}{1800}-\frac{t^{6} u^{4}}{1536}-\frac{3023393 t}{604800000}+\frac{t^{5} \lambda u^{2}}{80} \\
& -\frac{t^{8}}{720000}-\frac{t^{7}}{420000}+\frac{47 t^{6}}{8640000}+\frac{7 t^{5}}{1200000}-\frac{t^{2} u^{2}}{8}+\frac{16787 t u^{2}}{806400} \\
& +\frac{71971 t \lambda}{72000}-\frac{4001 u t}{4000}+u+\frac{t \lambda u^{2}}{320},
\end{aligned}
$$

for all $t \in[0,1], u \in U$, and $\lambda \in[-0.5,0.5]$.

The approximate solutions of the second approximate determining equation are $u=0.2016501379, \lambda=0.2016555796$. So the first and the second components of the second approximation have the form

$$
\begin{aligned}
x_{21}(t)= & 0.001666666667 t^{4}-0.27615755 \cdot 10^{-4} t^{3}-0.3289188868 \cdot 10^{-2} t^{2} \\
& -0.1+0.2016501380 t
\end{aligned}
$$

and

$$
\begin{aligned}
x_{22}(t)= & 0.2016501379-0.82847265 \cdot 10^{-4} t^{2}-0.61642052 \cdot 10^{-6} t^{4} \\
& +0.2210884634 \cdot 10^{-3} t^{3}-0.62 \cdot 10^{-10} t-0.1388888889 \cdot 10^{-5} t^{8} \\
& +0.39451079 \cdot 10^{-7} t^{7}+0.36543683 \cdot 10^{-5} t^{6}-0.1344879254 \cdot 10^{-3} t^{5} .
\end{aligned}
$$




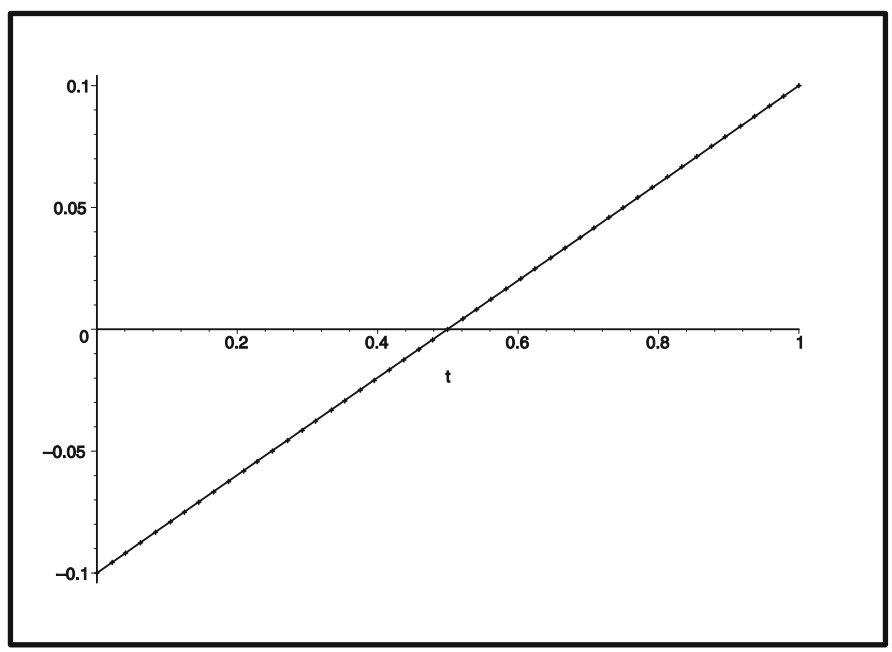

Figure 1. The first components of the exact solution (solid line) and its second approximation (drawn with dots).

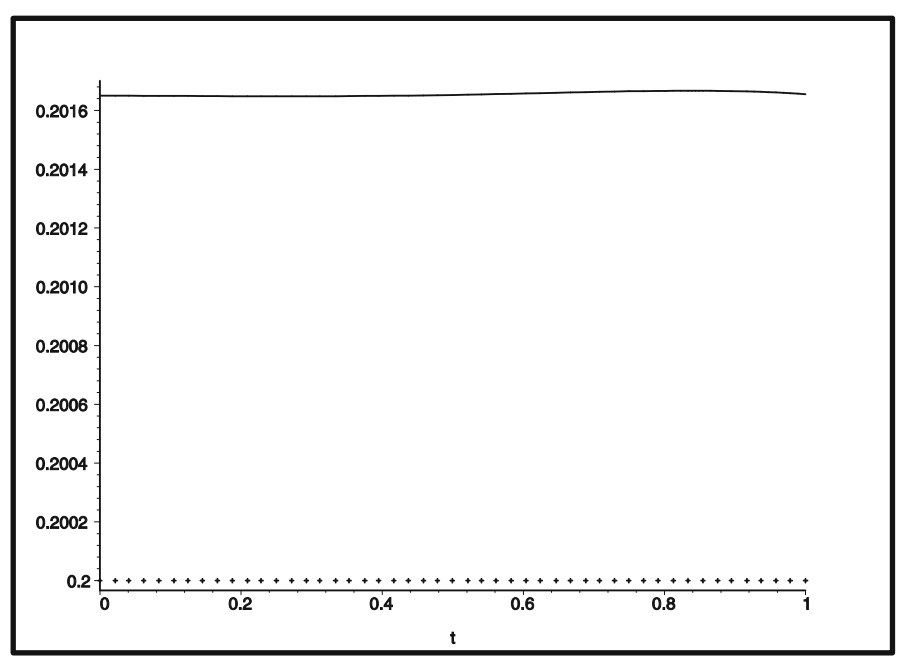

Figure 2. The second components of the exact solution (solid line) and its second approximation (drawn with dots).

As it is seen from Figures 1 and 2 presented above, the graph of the exact solution almost coincides with those of its second approximations. For example, the error of the second approximation (i.e., the uniform deviation of the first 


\section{PARAMETRIZATION FOR SOME BVPS OF INTERPOLATION TYPE}

approximation from the exact solution) admits the estimates

$$
\left|x_{1}^{*}(t)-x_{21}(t)\right| \leq 0.2 \cdot 10^{-3} ; \quad x_{2}^{*}(t)-x_{22}(t) \mid \leq 0.2 \cdot 10^{-2} .
$$

The error for the third approximation is

$$
\left|x_{1}^{*}(t)-x_{31}(t)\right| \leq 0.3 \cdot 10^{-5} ; \quad\left|x_{2}^{*}(t)-x_{32}(t)\right| \leq 0.11 \cdot 10^{-4},
$$

and the error for the fourth approximation is

$$
\left|x_{1}^{*}(t)-x_{41}(t)\right| \leq 0.25 \cdot 10^{-6} ; \quad\left|x_{2}^{*}(t)-x_{42}(t)\right| \leq 0.12 \cdot 10^{-5} \text {. }
$$

\section{REFERENCES}

[1] GOMA, I. A.: Method of successive approximations in a two-point boundary problem with parameter, Ukrainian Math. J. 29 (1977), No. 6, 594-599.

[2] GOMA, I. A.: On the theory of the solutions of a boundary value problem with a parameter, Azerbaĭdžan. Gos. Univ. Učen. Zap. Ser. Fiz.-Mat. Nauk 1 (1976), 11-16.

[3] HOSABEKOV, O.: Sufficient conditions for the convergence of the Newton-Kantorovič method for a boundary value problem with a parameter, Dokl. Akad. Nauk Tadžik. SSR 16 (1973), No. 8, 14-17.

[4] KURPEL, N. S.-MARUSJAK, A. G.: A multipoint boundary value problem for differential equations with parameters, Ukrain. Mat. Zh. 32 (1980), No. 2, 223-226. (In Russian)

[5] LUCHKA, A. YU.: Application of iterative processes to boundary value problems for differential equations with parameters, Dokl. Akad. Nauk Ukr., Ser. A 1989 (1989), No. 10, $22-27$.

[6] LUCHKA, A. YU.: Projection-Iteration Methods for the Solution of Differential and Integral Equations. (Proektsionno-Iterativnye Metody Resheniya Differentsialnykh i Integral'nykh Uravnenij.), Naukova Dumka, Kiev, 1980.

[7] AKHMEDOV, K. T.-SVARICHEVSKAYA, N. A.-YAGUBOV, M. A.: Approximate solution of a two-point boundary value problem with a prameter by the method of averaging functional corrections, Dokl. Akad. Nauk Azerbaidzhana 29 (1973), No. 8, 3-7.

[8] LUCHKA, A. YU.: The Method of Averaging Functional Corrections. Theory and Applications. Translated from the Russian by Scripta Technica, Inc., Academic Press, New York, 1965.

[9] LUCHKA, A. YU.-ZAKHARIICHENKO, YU. O.: Investigation of systems of differential equations with parameters under impulse conditions and with restrictions, Neliniini Kolyvannya 3 (2000), No. 2, 218-225.

[10] SAMOILENKO, A. M.-LUCHKA, A. YU.-LISTOPADOVA, V. V.: Application of iterative processes to a boundary value problem for a system of differential equations with impulse action and with parameters, Dokl. Akad. Nauk Ukr. 1994 (1994), No. 2, 15-20.

[11] LUCHKA, A. YU.: Projection-Iteration Methods. (Proektsionno-iterativnye metody). Naukova Dumka, Kiev, 1993.

[12] GAINES, R. E.-MAWHIN, J. L.: Coincidence Degree, and Nonlinear Differential Equations. Lecture Notes in Math., Vol. 568, Springer-Verlag, Berlin, 1977.

[13] FEČKAN, M.: Parametrized singular boundary value problems, J. Math. Anal. Appl. 188 (1994), 417-425.

[14] FEČKAN, M.: Parametrized singularly perturbed boundary value problems, J. Math. Anal. Appl. 188 (1994), 426-435. 


\title{
MIKLÓS RONTÓ - NATALIA SHCHOBAK
}

[15] KELLER, H. B.: Numerical Methods for Two-Point Boundary-Value Problems. Dover Publ., Inc., New York, 1992.

[16] ASCher, U. M.-MATtheiJ, R. M. M.-RUSSELL, R. D.: Numerical Solution of Boundary Value Problems for Ordinary differential Equations. in: Classics Appl. Math., Vol. 13, SIAM, Philadelphia, 1995.

[17] ABRAMOV, A. A.-UL'YANOVA, V. I.-YUKHNO, L. F.: A method for solving the multiparameter eigenvalue problem for certain systems of differential equations, Comput. Math. Math. Phys. 40 (2000), 18-26.

[18] SAMOILENKO, A. M.-RONTO, N. I.: Numerical-analytic Methods in the Theory of Boundary Value Problems for Ordinary Differential Equations. Naukova Dumka, Kiev, 1992.

[19] RONTÓ, M.: On numerical-analytic method for BVPs with parameters, Publ. Univ. Miskolc Ser. D, Nat. Sci., Math. 36 (1996), 125-132.

[20] RONTÓ, M.: On some existence results for parametrized boundary value problems, Publ. Univ. Miskolc Ser. D Nat. Sci. Math. 37 (1997), 95-103.

[21] RONTO, M.-SAMOILENKO, A. M.: Numerical-Analytic Methods in the Theory of Boundary-Value Problems. World Scientific Publishing Co. Inc., River Edge, NJ, 2000.

[22] RONTO, A.-RONTÓ, M.: On the investigation of some boundary value problems with non-linear conditions, Math. Notes (Miskolc) 1 (2000), 43-55.

[23] RONTO, A.-RONTÓ, M.: A note on the numerical-analytic method for nonlinear twopoint boundary-value problems, Nonlinear Oscil. 4 (2001), 112-128.

[24] RONTÓ, M.: On non-linear boundary value problems containing parameters, Arch. Math. (Brno) 36 (2000), 585-593.

[25] RONTÓ, M.: On the investigation of parametrized non-linear boundary value problems, in: Proc. of the 3rd World Congress of Nonlinear Analysts, Part 7, Catania, 2000, Nonlinear Anal., Vol. 47, 2001, pp. 4409-4420.

[26] RONTÓ, M.-SHCHOBAK, N.: On the numerical-analytic investigation of parametrized problems with nonlinear boundary conditions, Nonlinear Oscil. 6 (2003), 482-510.

[27] RONTO, M.-SHCHOBAK, N.: On parametrized problems with non-linear boundary conditions, in: Proc. of the 7th Colloq. on the Qualitative Theory of Differential Equations, No. 20, pp. 25 (electronic), Proc. Colloq. Qual. Theory Differ. Equ., Vol. 7, Electron. J. Qual. Theory Differ. Equ., Szeged, 2004.

[28] RONTO, A. N.-RONTO, M.-SHCHOBAK, N. M.: On the parametrization of threepoint nonlinear boundary value problems, Nonlinear Oscil. (7) (2004), 384-402.

Received September 12, 2008

\author{
Department of Analysis \\ University of Miskolc \\ H-3515 Miskolc-Egyetemvaros \\ HUNGARY \\ E-mail: matronto@gold.uni-miskolc.hu \\ Faculty of Mathematics \\ Uzhhorod National University \\ Pidhirna str. 46 \\ Uzhhorod, 88000 \\ UKRAINE \\ E-mail: natasha.shchobak@gmail.com
}

